\title{
Trefftz collocation for frequency domain elastodynamic problems
}

\author{
V. M. A. Leitão ${ }^{1}$, B. Sensale ${ }^{2}$ \& B. S. Rodriguez ${ }^{2}$ \\ ${ }^{I}$ DECivil-ICIST, Instituto Superior Técnico, TULisbon, Portugal \\ ${ }^{2}$ Facultad de Ingeniería, Universidad de la República, Uruguay
}

\begin{abstract}
A Trefftz collocation method is proposed for the analysis of two-dimensional elastodynamic problems subjected to steady-state time-harmonic loads. Trefftz methods are characterized by the use of a superposition of a number of actual solutions of the homogeneous part of governing equations, the so-called $\mathrm{T}(\mathrm{Trefftz})$-functions. This leads to high quality approximations where the unknowns are the weights of each of the T-functions considered. The unknowns are found by matching the approximations to the boundary conditions by following a standard collocation approach.

The numerical implementation of the method is briefly described and results are obtained for two anti-plane elastodynamic problems. The results compare quite favourably to other results available in the literature whereby Galerkin and collocation boundary element methods were used.
\end{abstract}

Keywords: Trefftz method, elastodynamics, collocation.

\section{Introduction}

The Trefftz method (Trefftz [1]), consists essentially in building an approximation by resorting to a set of actual solutions of the homogeneous system of partial differential equations that governs the boundary value problem. As the homogeneous part of the equations is well taken care of, all that remains to be done is the (approximate) enforcement of the boundary conditions. The traditional way to achieve this is by collocation (Leitão [2, 3], Sensale and Rodriguez [4]). This means selecting a set of (boundary) points and forcing the approximation to satisfy, locally, the boundary conditions.

The use of the Trefftz concept is not restricted to boundary solution methods. Galerkin approaches typical of the conventional formulations of the finite 
element methods may also be used to implement the Trefftz method (Freitas [5]) but this will not be addressed here.

The solutions for the homogeneous differential equations of a given problem are, in the Trefftz approach, obtained in the form of an infinite series of regular functions centered at a given point and well defined everywhere. Solutions for the homogeneous differential equations may also be obtained in the form of fundamental (singular) solutions. This is the approach followed by the Boundary Element Method (BEM) (see Brebbia et al. [6]) and by the Method of Fundamental Solutions (MFS) (see Fairweather and Karageorghis [7]).

In what follows the "Trefftz method" is characterized by the following: 1) each function of the approximation basis is a non-singular term of an infinite series (although singular functions may be added to the basis when necessary); 2) the collocation approach is used (even though the more general setting of weighted least squares may also be used).

The range and scope of applications of the Trefftz method, be it the collocation or the Galerkin-based approaches, has seen a strong increase in the last decade. Reviews on the subject may be found in Kita and Kamya [8], Jirousek and Zielinski [9] and Li et al. [10].

In this work the Trefftz collocation method is applied to the analysis of twodimensional elastodynamic problems subjected to steady-state time-harmonic loads. As will be later shown this problem may be simplified in a way that leads to the Helmholtz equation, which is the equation that models the scattering of acoustic waves. Trefftz methods are particularly suited for this type of problems because, contrary to the finite element method and, in a certain way, the boundary element method the discretization requirements are not dependent on the wavelength considered. Trefftz collocation methods have essentially been applied to the case of acoustics, that is, the scalar Helmholtz case (see Li [11]). Galerkin type Trefftz methods have been applied to the acoustic case (see Pluymers et al. [12], Cheung et al. [13]) but also to the elastodynamic case (Freitas and Cismasiu [14]) amongst others.

The numerical validation of the proposed method is achieved by the application to a set of anti-plane elastodynamic problems for which analytic (Kitahara [15]) as well as other numerical solutions are available (Dominguez [16] and Perez-Gavilan and Aliabadi [17].)

\section{The Navier equations}

The motion, with respect to time, of homogeneous, isotropic, linear elastic bodies is described by the Navier equations. These are obtained by appropriately replacing the compatibility (kinematic) description (and the strains are assumed to be infinitesimal for the sake of simplicity) into the constitutive relationship followed by the substitution into the equilibrium (static) equation. The resulting equation, written in terms of displacements, has to be satisfied everywhere in the domain together with the satisfaction of the boundary and initial conditions of the problem. 
A starting assumption that will be made here is that the main variables of the problem vary harmonically in time, that is, all variables depending on time are functions of $\sin \omega t$ and $\cos \omega t$ where $\omega$ is the (angular) frequency. This assumption allows the problem to be stated in the frequency domain by means of a Fourier transform.

When dealing with problems that may exhibit some form of damping it is convenient to resort to the more general setting of viscoelasticity, that is, to the case when the material characteristics vary with time. In this case the following two independent complex-valued Lamé type coefficients may be obtained:

$$
\begin{aligned}
& \mu(\omega)=\frac{1}{2(1+v)} E^{*}(\omega) \\
& \lambda(\omega)=\frac{v}{(1+v)(1-2 v)} E^{*}(\omega)
\end{aligned}
$$

where $E^{*}(\omega)$ is the complex modulus and $v$ is the time independent (for the sake of simplicity) Poisson coefficient.

When damping effects are present these Lamé type coefficients take the form:

$$
\begin{aligned}
& \mu=\operatorname{Re}[\mu(\omega)]\left(1+2 i \beta_{\mu}\right) \\
& \lambda=\operatorname{Re}[\lambda(\omega)]\left(1+2 i \beta_{\lambda}\right)
\end{aligned}
$$

By expressing the constants in this convenient form there are no differences between the governing equations for the elastic or the viscoleastic case when treating time-harmonic problems in the frequency domain.

In the expressions above $\beta_{\mu}$ and $\beta_{\lambda}$ are damping factors. It is usual to consider $\beta=\beta_{\mu}=\beta_{\lambda}$, and refer to $\beta$ as being the viscous damping factor.

\section{Formulation of the elastodynamic equation}

The general form of the elastodynamic governing equation for viscoelastic materials in the time domain is (Christensen [18]):

$$
\begin{aligned}
c_{S}^{2}(t, \tau) * \Delta \vec{u}(X, t)+\left(c_{P}^{2}(t, \tau)\right. & \left.-c_{S}^{2}(t, \tau)\right) * \nabla(\nabla \cdot \vec{u}(X, t))+ \\
& +\vec{b}(X, t)=\overrightarrow{\vec{u}}(X, t), \quad \forall X \in \Omega
\end{aligned}
$$

where $\vec{b}(X, t)$ is the body force load vector, and

$$
\begin{aligned}
c_{S}^{2}(t, \tau) * & =\frac{1}{2(1+v) \rho} R_{E}(t, \tau) * \\
c_{P}^{2}(t, \tau) * & =\frac{1-v}{(1+v)(1-2 v) \rho} R_{E}(t, \tau) *
\end{aligned}
$$


are, respectively, the square of the S-wave velocity and the square of the P-wave velocity in the viscoelastic case, $v$ is the Poisson coefficient, $\rho$ is the density of the material and $R_{E}(t, \tau)$ is its relaxation function.

For the sake of simplicity let us assume that the boundary conditions (displacements $\vec{u}(X, t)$ and tractions $\vec{p}(X, t))$ vary harmonically in time as follows:

$$
\begin{aligned}
& \vec{u}(X, t)=\vec{u}_{o}(X) e^{i \omega_{o} t}, \quad \forall X \in \partial \Omega_{u} \\
& \vec{p}(X, t)=\vec{p}_{o}(X) e^{i \omega_{o} t}, \quad \forall X \in \partial \Omega_{p}
\end{aligned}
$$

and that the body forces are not present.

In these conditions, that is, for time-harmonic problems, a description of the governing equation in the frequency domain is achieved by applying the Fourier transform to the general equation in time. The result may be written as:

$$
\begin{array}{r}
\bar{c}_{S}^{2}(\omega) \Delta \overrightarrow{\vec{u}}(X, \omega)+\left(\bar{c}_{P}^{2}(\omega)-\bar{c}_{S}^{2}(\omega)\right) \nabla(\nabla \cdot \overrightarrow{\bar{u}}(X, \omega))- \\
-\omega^{2} \overrightarrow{\bar{u}}(X, \omega)=0, \quad \forall X \in \Omega
\end{array}
$$

where the bar over the displacement and traction denotes the transformed variables and:

$$
\begin{aligned}
& \bar{c}_{S}^{2}(\omega)=\frac{1}{2(1+v) \rho} E^{*}(\omega) \\
& \bar{c}_{P}^{2}(\omega)=\frac{1-v}{(1+v)(1-2 v) \rho} E^{*}(\omega)
\end{aligned} .
$$

The boundary conditions now read:

$$
\begin{array}{ll}
\overrightarrow{\vec{u}}(X, \omega)=\vec{u}_{o}(X) \delta\left(\omega-\omega_{o}\right), & \forall X \in \partial \Omega_{u} \\
\vec{p} & (X, \omega)=\vec{p}_{o}(X) \delta\left(\omega-\omega_{o}\right), \quad \forall X \in \partial \Omega_{p}
\end{array}
$$

The above description is a three-dimensional one. The displacement field has, therefore, three components:

$$
\vec{u}(X, t)=u_{1} \vec{e}_{1}+u_{2} \vec{e}_{2}+u_{3} \vec{e}_{3}
$$

In this work the three-dimensional field will be restricted to the anti-plane case only.

The geometry of the anti-plane case is a two-dimensional one and displacements only occur in the $x_{3}$ direction, that is:

$$
\begin{aligned}
& u_{1}=u_{2}=0 \\
& u_{3}=u_{3}\left(x_{1}, x_{2}, t\right)
\end{aligned}
$$

The only non-null components of the stress tensor are $\sigma_{13}$ and $\sigma_{23}$.

Due to these simplifications $u_{3}$ is governed by a simplified version of the elastodynamic equation, the scalar wave equation: 


$$
\bar{c}_{S}^{2} \Delta \bar{u}_{3}\left(x_{1}, x_{2}, \omega\right)+\omega^{2} \bar{u}_{3}\left(x_{1}, x_{2}, \omega\right)=0
$$

where only the S-wave velocity is considered.

\section{Helmholtz decomposition}

Helmholtz theorem (see Pak and Eskandari-Ghadi [19]) states that any twice differentiable vector field $\vec{u}$ may be, uniquely, decomposed into a sum of two field vectors, an irrotational one $\vec{u}_{P}$ not present in the anti-plane case) and a solenoidal one $\vec{u}_{S}$.

The application of the Helmholtz decomposition to the Navier equation in the frequency domain as shown above leads to:

$$
\begin{aligned}
& \bar{c}_{S}^{2} \Delta\left[\vec{u}_{P}(\omega)+\vec{u}_{S}(\omega)\right]+\left(\bar{c}_{S}^{2}-\bar{c}_{P}^{2}\right) \nabla\left(\nabla \cdot\left[\vec{u}_{P}(\omega)+\vec{u}_{S}(\omega)\right]\right)+ \\
& +\omega^{2}\left[\vec{u}_{P}(\omega)+\vec{u}_{S}(\omega)\right]=0 \\
& \Delta \vec{u}_{P}(\omega)=\nabla\left(\nabla \cdot \vec{u}_{P}(\omega)\right)-\operatorname{rot}\left(\operatorname{rot} \vec{u}_{P}(\omega)\right)=\nabla\left(\nabla \cdot \vec{u}_{P}(\omega)\right) \\
& \Delta \vec{u}_{S}(\omega)=\nabla\left(\nabla \cdot \vec{u}_{S}(\omega)\right)-\operatorname{rot}\left(\operatorname{rot} \vec{u}_{S}(\omega)\right)=-\operatorname{rot}\left(\operatorname{rot} \vec{u}_{S}(\omega)\right) \\
& \bar{c}_{P}^{2} \Delta \vec{u}_{P}(\omega)+\bar{c}_{S}^{2} \Delta \vec{u}_{S}(\omega)+\omega^{2} \vec{u}_{P}(\omega)+\omega^{2} \vec{u}_{S}(\omega)=0
\end{aligned}
$$

Looking only at the solenoidal field and considering a vector potential $\vec{\psi}$

$$
\vec{\psi}=\psi_{1} \vec{e}_{1}+\psi_{2} \vec{e}_{2}+\psi_{3} \vec{e}_{3}
$$

the following form is obtained:

$$
\begin{aligned}
\vec{u}_{S}=\operatorname{rot} \vec{\psi} \Rightarrow \operatorname{rot}[ & \left.\bar{c}_{S}^{2} \Delta \vec{\psi}+\omega^{2} \vec{\psi}\right]=0 \\
& \Rightarrow \bar{c}_{S}^{2} \Delta \vec{\psi}+\omega^{2} \vec{\psi}=0
\end{aligned}
$$

where, for the anti-plane case, only the $\psi_{3} \vec{e}_{3}$ matters.

\section{The Trefftz collocation approach}

The starting point in any Trefftz approach is the approximation of the variable of interest, in this case the displacement field, by a superposition of an infinite number of functions each of them solution of the homogeneous governing equation, that is:

$$
\vec{u}_{d}(X, \omega)=\sum_{i=1}^{N} \vec{a}_{i} \vec{u}_{i}^{*}(X, \omega)
$$

where the $\vec{a}_{i} \in \mathbb{R}^{3}$ represent the unknowns, that is, the weights affecting each of the $\vec{u}_{i}^{*}$ functions. The full series of functions is usually referred to as the T- 
complete series in the sense that any solution of the actual problem under consideration is included in the space spanned by the T-complete series.

Applying the collocation approach, at selected $X_{i}$ points located at the boundary $\partial \Omega$, the following system arises:

$$
\begin{array}{ll}
\vec{u}_{d}\left(X_{i}\right)=\vec{u}\left(X_{i}\right) & \forall X_{i} \in \partial \Omega_{u} \\
\vec{p}_{d}\left(X_{i}\right)=\vec{p}\left(X_{i}\right) & \forall X_{i} \in \partial \Omega_{p}
\end{array}
$$

where the left-hand side contains the Trefftz approximation and the right-hand side represents the (known) boundary conditions.

\section{Trefftz functions for the anti-plane case}

The governing equation is of the scalar Helmholtz type. The displacement component of interest in the anti-plane case is described by the following $\mathrm{T}$ complete series (Qin [20]):

- for a bounded region:

$$
\begin{aligned}
\vec{u}_{d}(X)=u_{3}(X) & =c_{0} J(k r)+ \\
& +\sum_{m=1}^{N^{*}}\left[c_{2 m} J_{m}(k r) \cos (m \theta)+c_{2 m+1} J_{m}(k r) \sin (m \theta)\right]
\end{aligned}
$$

- for an unbounded region:

$$
\begin{aligned}
\vec{u}_{d}(X)=u_{3}(X) & =c_{0} J(k r)+ \\
& +\sum_{m=1}^{N^{*}}\left[c_{2 m} H_{m}(k r) \cos (m \theta)+c_{2 m+1} H_{m}(k r) \sin (m \theta)\right]
\end{aligned}
$$

The surface stress vector at point $X$ of the boundary of normal $\vec{n}(X)=n_{1} \vec{e}_{1}+n_{2} \vec{e}_{2}$ is:

$$
\vec{p}_{d}(X)=p_{3}(X)=\frac{E^{*}(\omega)}{2(1+v)}\left(\frac{\partial u_{3}(X)}{\partial x_{1}} n_{1}+\frac{\partial u_{3}(X)}{\partial x_{2}} n_{2}\right)
$$

\section{Calculation of the natural frequencies}

The above representations can be immediately applied to obtain the displacements and stresses but one aspect of interest in this type of elastodynamic problems is the calculation of the natural or resonance frequencies.

The procedure used in this work to determine the natural frequencies starts by evaluating a measure (a norm) of the solution of the problem at a given set of domain points for a range of frequencies. 
A search method, in this case the Nelder-Mead Simplex Method, is then applied to fine tune the capture of the natural frequencies as those for which the norm of the solution takes the highest value.

\section{Numerical results}

The set of examples tested were previously analysed in the works of Domínguez [16], Kitahara [15] and Perez-Gavilan [17].

In all cases the natural frequencies points are calculated and compared to analytic results and to the numerical results shown in the above references.

\subsection{Square region under uniform anti-plane shear}

A $6 \mathrm{~m} \times 6 \mathrm{~m}$ square region, clamped on one side, free on all others, is subjected to a uniform anti-plane shear load $p=100 \mathrm{~N} / \mathrm{m}^{2}$ on the opposite side.

The material properties are: shear modulus $\mu=10^{6} \mathrm{~N} / \mathrm{m}^{2}$; specific mass $\rho=100 \mathrm{Kg} / \mathrm{m}^{3}$; damping coefficient $\beta=0.05$.

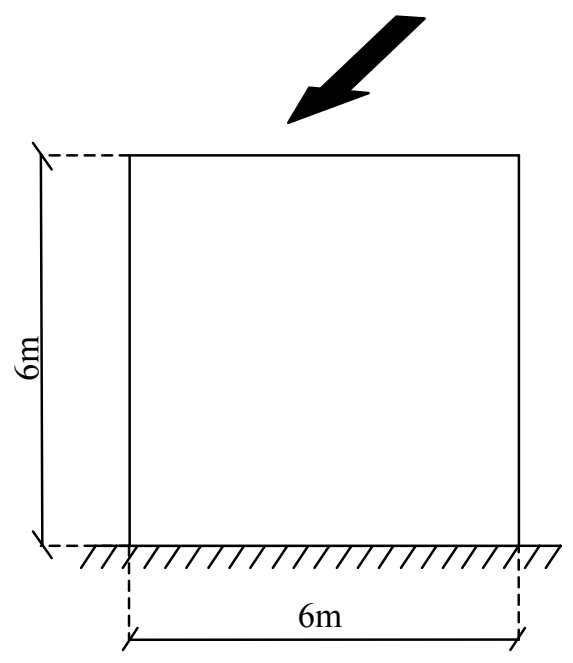

Figure 1: $\quad$ Square region under uniform shear.

An analytical solution of this square domain (in fact, a one dimensional) problem exists for the case of perfectly elastic material, that is, for the case of a material with null damping. The resonance frequencies are $\omega_{n}=(2 n-1) \pi c_{S} /(2 a)$ for a $a \times a$ square region. In this case, for which the shear-wave velocity is $c_{S}=100 \mathrm{~m} / \mathrm{s}$, and $a=6$ the first frequencies are $26.18 \mathrm{~s}^{-1}, 78.54 \mathrm{~s}^{-1}, 130.90 \mathrm{~s}^{-1}, \ldots$ 
The differences when a 5\% material damping is considered are negligible. These values, shown in figure 2 for the square region under uniform shear, are exactly reproduced by the present method.

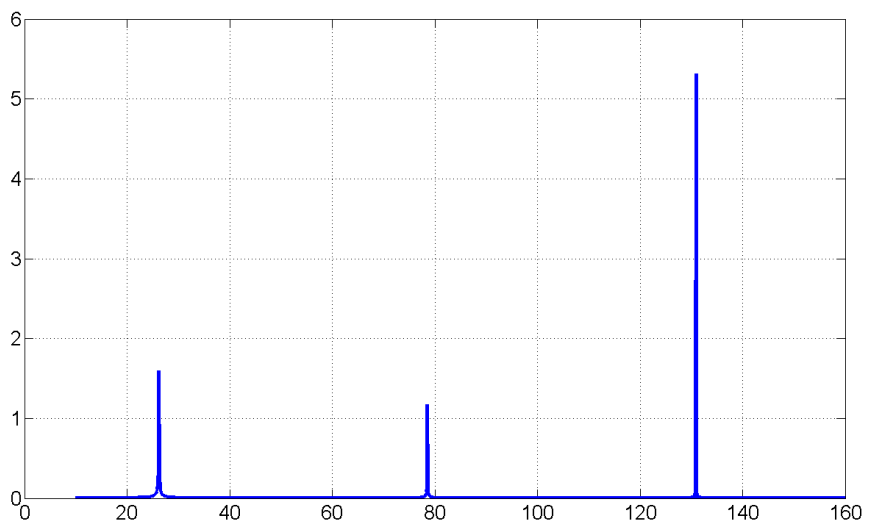

Figure 2: $\quad$ Norm of the solution for a range of frequencies.

\subsection{Infinite cylinder under uniform axial load/displacement on the surface}

This is an anti-plane problem now with a circular cross-section of radius $6 \mathrm{~m}$. The material properties are the same as for the previous examples.

The boundary conditions are:

- unit uniform axial displacement;

- uniform axial load on the surface $p=100 \mathrm{~N} / \mathrm{m}^{2}$.

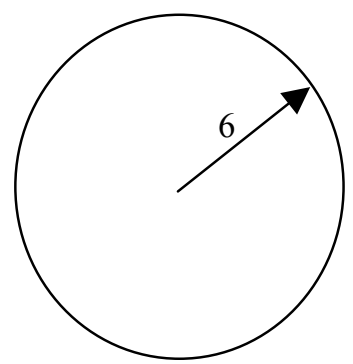

Figure 3: Cross-section of infinite cylinder under uniform load.

The first two resonance frequencies for the case of unit uniform axial displacement, $\omega=40.08,92.00$, match the analytical ones.

And the same for the case of uniform axial load, the first two resonance frequencies, $\omega=63.87,116.93$, match the analytical ones. 

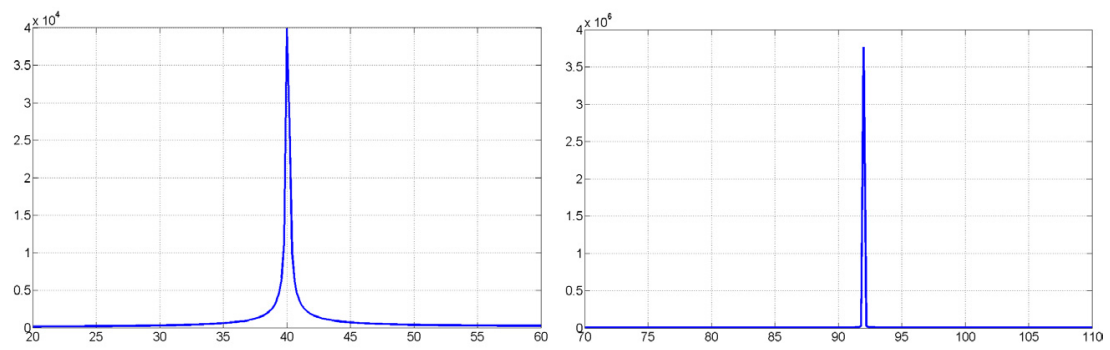

Figure 4: $\quad$ First two resonance frequencies for the case of unit uniform axial displacement.
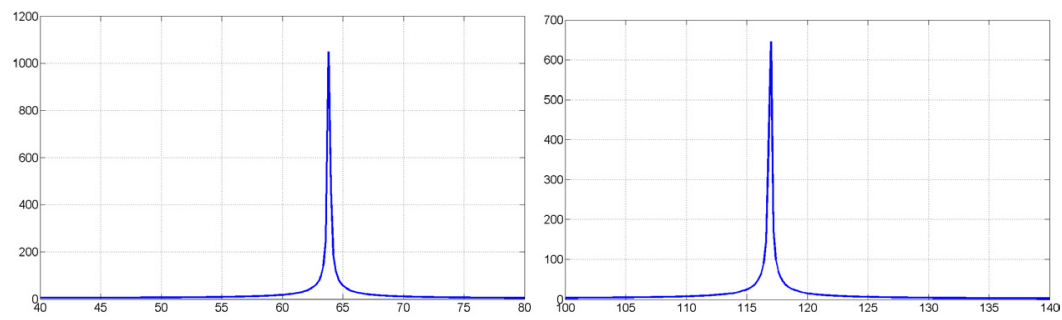

Figure 5: $\quad$ First two resonance frequencies for the case of unit uniform axial load.

\section{Conclusions}

In this work a Trefftz collocation method has been presented and applied to the analysis of elastodynamic problems in the frequency domain.

The method relies on the use of actual solutions of the homogeneous governing equation which are seen to be scalar and vector potentials satisfying the Helmholtz equation. A solution is found by superposing an appropriate number of terms of the infinite series representing the solution of the homogeneous governing equation followed by the enforcement of the boundary conditions using the collocation approach.

The results obtained in this manner for a set of representative problems compare very well with analytical and numerical results available in the literature thus showing the potential of this technique.

\section{References}

[1] Trefftz, E., (1926), “Ein Gegenstuck zum Ritzschen Verharen”, paper presented at 2nd Int. Cong. Appl. Mech., Zurich, pp. 131-137. 
[2] Leitão, V.M.A., (1997), "On the implementation of a multi-region Trefftzcollocation formulation for 2-D potential problems", Engineering Analysis with Boundary Elements, Vol. 20, pp. 51-61.

[3] Leitão, V.M.A., (1998) "Application of multi-region Trefftz-collocation to fracture mechanics", Engineering Analysis with Boundary Elements, Vol 22, pp. 251-256.

[4] Sensale, B., Sensale Rodriguez, B., (2007), "The Trefftz Boundary Method in Viscoelasticity", CMES: Computer Modeling in Engineering \& Sciences, Vol.20, $\mathrm{N}^{\circ} 1$, pp. 21-33.

[5] Freitas, J.A.T., (1998), "Formulation of elastostatic hybrid-Trefftz stress elements", Comp. Meth. Appl. Mech. Eng. 153, pp. 127-151.

[6] Brebbia, C.A., Telles, J.C.F., Wrobel, L.C., (1984), Boundary Element Techniques. Theory and Applications in Engineering, Springer-Verlag, Berlin.

[7] Fairweather, G., Karageorghis, A., (1998), "The method of fundamental solutions for elliptic boundary value problems", Adv. Comp. Math. 9, pp. 69-95.

[8] Kita, E., Kamiya, N., (1995), "Trefftz method, An overview”, Adv. Engrg. Softw., v24, pp. 3-12.

[9] Jirousek, J., Zielinski, A.P., (1997), "Survey of Trefftz-type element formulations", Computers \& Structures, 63(2), pp. 225-242.

[10] Li, Z.C., Lu, T.T., Hu, H.Y., Cheng, A., (2008), Trefftz and Collocation Methods, WIT Press.

[11] Li, Z.C., (2008), "The Trefftz method for the Helmholtz equation with degeneracy", Applied Numerical Mathematics, Vol. 58, pp. 131-159.

[12] Pluymers, B., van Hal, B., Vandepitte, D., Desmet, W., (2007), "Trefftz.based methods for time-harmonic acoustics", Arch. Comput. Methods Eng., Vol. 14, pp. 343-381.

[13] Cheung, Y.K., Jin, W.G., Zienkiewicz, O.C., (1991), "Solution of Helmholtz equation by Trefftz method", International Journal For Numerical Methods in Engineering, Vol. 32, pp.63-78.

[14] Freitas, J.A.T., Cismasiu, C., (2003), "Hybrid-Trefftz displacement element for spectral analysis of bounded and unbounded media", International Journal of Solids and Structures, Vol.40, pp. 671-699.

[15] Kitahara, M., (1985), Applications of boundary integral equation methods to eigenvalue problems of elastodynamic and thin plates, Elsevier, Amsterdam.

[16] Dominguez, J., (1993), Boundary Elements in dynamics, Computational Mechanics Publication, Elsevier Applied Science, Amsterdam.

[17] Perez-Gavilán, J.J., Aliabadi, M.H., (2001), “A symmetric Galerkin boundary element method for dynamic frequency domain viscoelastic problems", Computers and Structures, Vol.79, pp.2621-2633

[18] Christensen, R.M., (1982), Theory of Viscoelasticity, An Introduction, $2^{\text {nd }}$ edn., Academic Press, New York. 
[19] Pak, R.Y.S., Eskandari-Ghadi, M., (2007), "On the completeness of a method of potentials in elastodynamics", Quarterly of Applied Mathematics Vol.LXV, N4, pp. 789-797.

[20] Qin, Q.H., (2000), The Trefftz Finite and Boundary Element Method, WIT Press, Southampton. 